\title{
Hard x-ray optics for the HEFT balloon-borne payload: prototype design and status
}

William W. Craig, Finn Erland Christensen, Todd R. Decker, Charles J. Hailey, Fiona A. Harrison, et al.

William W. Craig, Finn Erland Christensen, Todd R. Decker, Charles J. Hailey, Fiona A. Harrison, Randal M. Hill, Mario A. Jimenez-Garate, Peter H. Mao, Steven M. Schindler, "Hard x-ray optics for the HEFT balloon-borne payload: prototype design and status," Proc. SPIE 3445, EUV, X-Ray, and Gamma-Ray Instrumentation for Astronomy IX, (10 November 1998); doi:

10.1117/12.330269 


\title{
Hard X-ray Optics for the HEFT Balloon Borne Payload: Prototype Design \& Status
}

\author{
William W. Craig ${ }^{a}$, Finn E. Christensen ${ }^{b}$, Todd Decker ${ }^{a}$, Charles J. Hailey ${ }^{\mathrm{a}}$, Fiona A. Harrison ${ }^{\mathrm{c}}$, \\ Randall M. Hill ${ }^{\mathrm{a}}$, Mario Jimenez-Garate ${ }^{\mathrm{a}}$, Peter Mao ${ }^{\mathrm{c}}$, Steven Schindler ${ }^{\mathrm{c}}$ \\ ${ }^{a}$ Columbia Astrophysics Lab, Columbia University, 550 West $120^{\text {th }}$ Street New York, NY 10027 \\ ${ }^{b}$ Danish Space Research Institute, Juliane Maries Vej 30, DK-2100 Copenhagen, Denmark \\ ${ }^{\mathrm{c}}$ Space Radiation Laboratory, California Institute of Technology, MC220-47 Pasadena, CA 91125
}

\begin{abstract}
We report on the current status and performance of prototype hard X-ray optics we are producing for use on the High Energy Focusing Telescope (HEFT) experiment. The baseline substrates are thermally formed glass mirrors that are overcoated with multilayers to provide good performance throughout the $20-80 \mathrm{keV}$ bandpass. Progress made in the thermal forming process as well as in the multilayer performance has allowed production of optics that meet or exceed all HEFT requirements. We present metrology on the substrates and results from X-ray characterization. A novel mounting scheme for the individual telescope shells is currently being tested. If successful the mounting technique will produce a monolithic, extremely stiff and robust optic.
\end{abstract}

\section{INTRODUCTION}

\subsection{HEFT Requirements}

The HEFT balloon borne experiment, shown as Figure 1 below, contains a number of individual mirror modules that will focus X-rays in the 20-80 keV bandpass on to Cadmium Zinc Telluride (CZT) detectors 6 meters away. The payload is scheduled for first flight in 2001. The optics requirement is for a focused spot of $<5$ arcminutes HEW. The sensitivity of the experiment is increased significantly if the spot size can be decreased. The current goal for the hard $\mathrm{X}$-ray optics is a HEW of 1 arcminute. This goal for system performance leads to a requirement on individual shells, which should have 0.5 arcminute or better figure.

After successfully producing and examining the performance ${ }^{1}$ of Epoxy Replicated Aluminum Foils, the HEFT program has adopted, as a baseline, thermally formed glass substrates for the optics. Glass provides a number of advantages:

- Excellent intrinsic micro-roughness (3.5 $)$

- Multilayers can be deposited directly with good adhesion

- Manufacturing process is simple and high yield.

The material and process we describe here easily meets the HEFT requirements and has the potential of allowing the optics to exceed HEFT goals and meet goals for the Hard X-ray Telescope on the Constellation-X mission ${ }^{2}$.

\subsection{HEFT Design}

The HEFT optics are conical approximations to a Wolter-1 geometry. Fourteen individual modules are planned. Each module consists of 72 highly nested shells. The shells will be produced in sectors (quadrants or thirds) requiring thousands of individual shells (8064 if quadrants are used). This large number heavily favors procedures that simplify the manufacturing process. The program budget mandates that the optics be produced in a university laboratory. The shells are produced by thermal forming, then coated with a 'graded-d' spacing multilayer (Section 3), and mounted into a sector of a mirror module (see Section 4). 


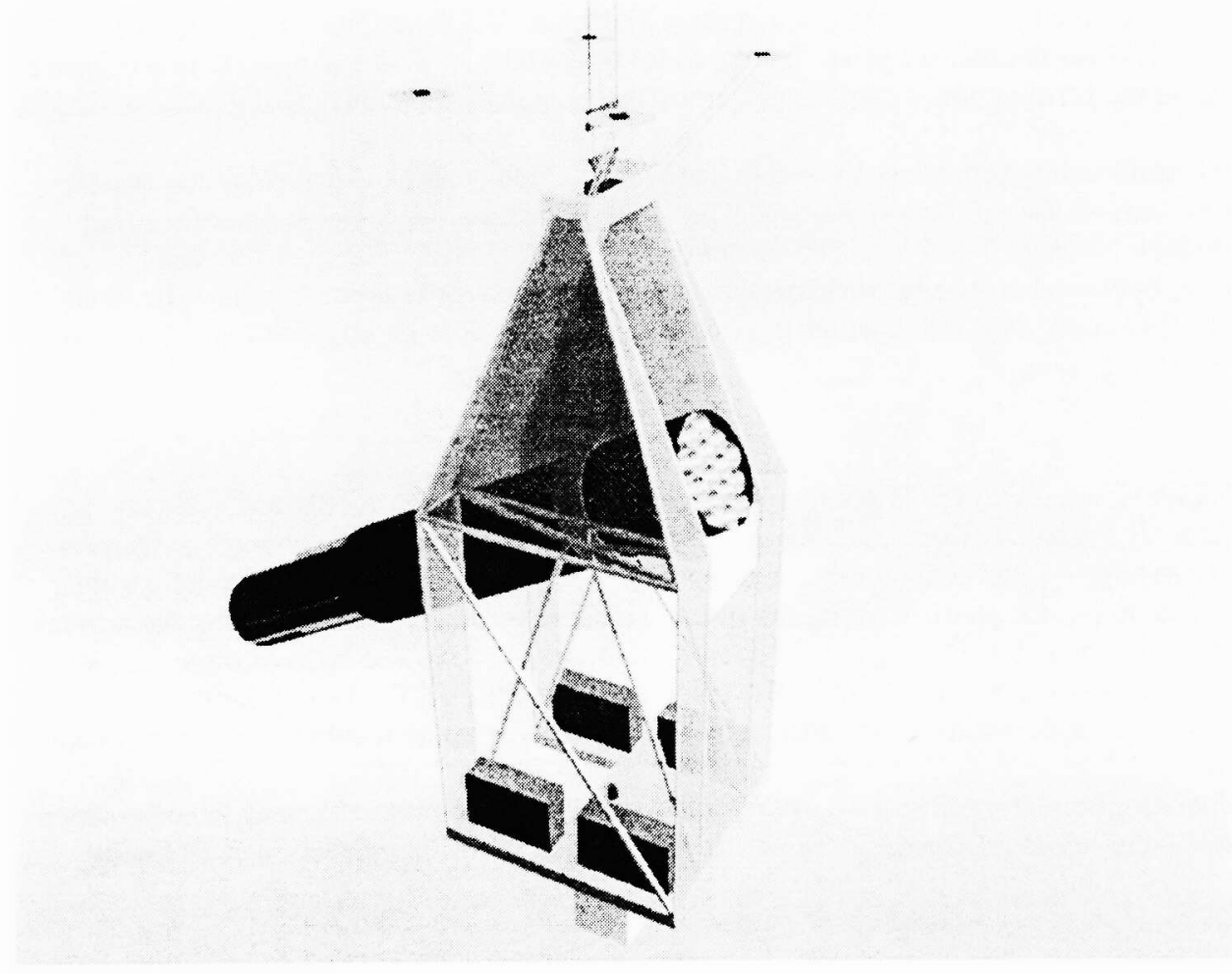

Figure 1: The HEFT payload design showing the 14 hard $\mathrm{x}$-ray optics modules. The telescope has a focal length of $6 \mathrm{~m}$.

\subsection{Prototype Design}

We are currently constructing a representative prototype sector that is intended to demonstrate performance and validate manufacturing processes before full-scale production of flight units is undertaken. This prototype contains 24 thermally formed glass substrates coated with the HEFT graded-d multilayer design mounted in a flight representative fixture. This prototype is intended to demonstrate both the mechanical and X-ray performance of the current HEFT optic design.

\section{SUBSTRATE DEVELOPMENT}

Progress since our initially reported ${ }^{1}$ results on substrate candidates has led us to baseline thermally formed glass over the primary alternative (Epoxy Replicated Aluminum Foils). The potential performance of the thermally formed glass as an X-ray substrate exceeds, by more than a factor of two, that of an ERAF based optic.

\subsection{Thermal Forming Procedure}

We use Desag AF-45 glass microsheet, which has excellent properties for an X-ray substrate (Section 2.2). This glass, produced by means of a process that avoids any mechanical contact with the surface, is extraordinarily strong and smooth at the level required by HEFT (or other hard/soft X-ray telescopes). The $300 \mu \mathrm{m}$ thick glass is formed into cylindrical mandrels and then mounted to produce the desired conical approximation to a Wolter 1 optic. We use quartz mandrels, into which the glass is slumped under gravity at temperatures above the softening point of the glass and below the annealing point. A silica based separation layer, placed between the glass and the quartz mandrel, facilitates the forming procedure and increases yield (but is not required to produce satisfactory results).

\subsubsection{Process Optimization}

Efforts over the past year have concentrated on development of a process that reliably and repeatedly produces HEFT quality optics. The primary hurdle in the development of thermally formed glass is the elimination of centimeter scale ripples (of amplitude $\sim$ few arcminutes) that can be produced during the process. We have found that these ripples can be eliminated by tight control of the forming process. A substantial improvement was made by modifications made to the commercial oven used in the forming process. These modifications included improved sealing procedures on the 
doors and the addition of formed ceramic structures in the interior of the oven which increase convective flow and buffer the heat while controlling radiative input from the oven heating elements. We found that these improvements increased both performance and yield for the formed glass. The goal for uniformity, $<+/-2.5$ degrees $\mathrm{C}$ in the forming region of the oven during the time the forming takes place is driven by the very steep dependence of glass viscosity on temperature.

Modifications to the ramp, soak and venting procedure have also been made. Each change is driven by the need to keep the glass in a uniform environment throughout the process while still maintaining acceptable manufacturing throughput. Each oven can produce between 3 and 5 substrates per day on a single shift. The ovens themselves are relatively inexpensive, two are in operation currently, with more planned once flight production begins. The yield appears to be greater than $70 \%$. The final yield will become clearer as the recently completed metrology equipment becomes a standard part of the forming process.

\subsubsection{Planned Changes}

The modifications that have been made to the thermal process have greatly improved both quality and yield and have established that HEFT optics can be produced. There are, however, a number of potential improvements to the process that are not implemented at this writing. Chief among these is a method to reduce or eliminate the gravity gradient implicit in the current passive technique. By gently rocking the quartz mandrel, or the oven itself, during the critical few moments of the forming process, the sides of the glass substrates can be made to see the same average gravity vector as the center. This uniformity allows better control of the velocity of the piece and reduces potential for stretching or distorting. Testing of possible designs and construction of a prototype apparatus to test this improvement is underway.

More advanced refinements, including direct modification of the radiative input to the glass, that may be necessary to achieve the tolerances of future $\mathrm{X}$-ray telescopes, are under investigation as part of the Constellation- $\mathrm{X}$ technology development program.

\subsection{Measurements}

We have firmly established that the glass substrates, both flat and thermally formed, have excellent X-ray properties at length scales below a millimeter. These scales are the most important as they measure intrinsic properties of the glass itself and are difficult to measure by any conventional mechanical or non-contact figure measurement method. Using total integrated scattering measurements, we have measured the glass on all length scales up to a millimeter. Figure 2

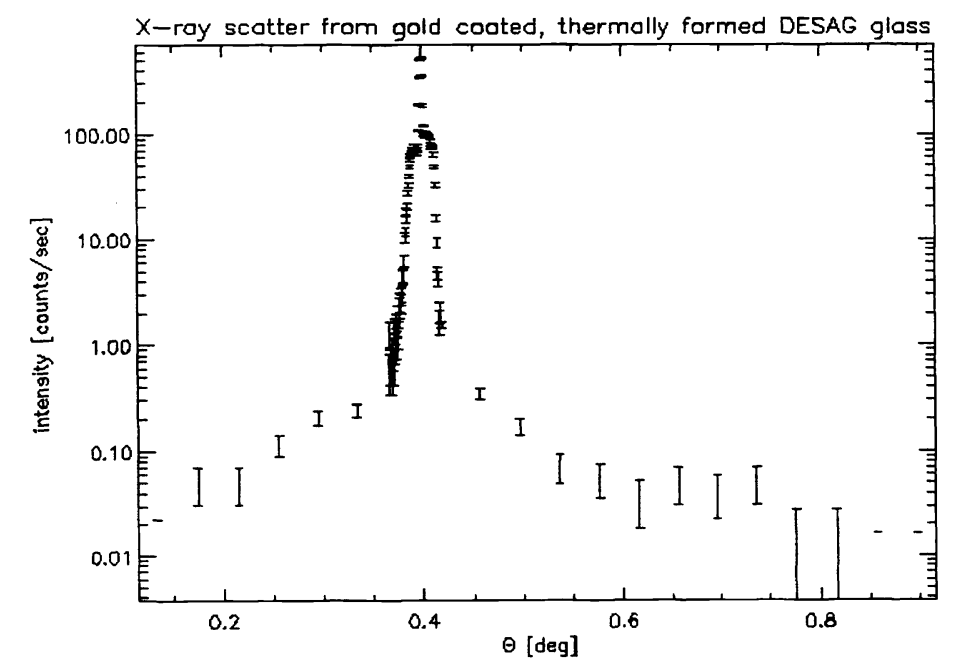

Figure 2: Total integrated scattering measurements made on a sample of thermally formed AF45 glass. The roughness derived is consistent with the $4 \AA$ measured on the glass before forming

shows the results from a piece of thermally formed AF-45 glass. The thermal forming process has no measurable effect on the roughness of the glass surface, which is consistent with $4 \AA$ before and after forming. 


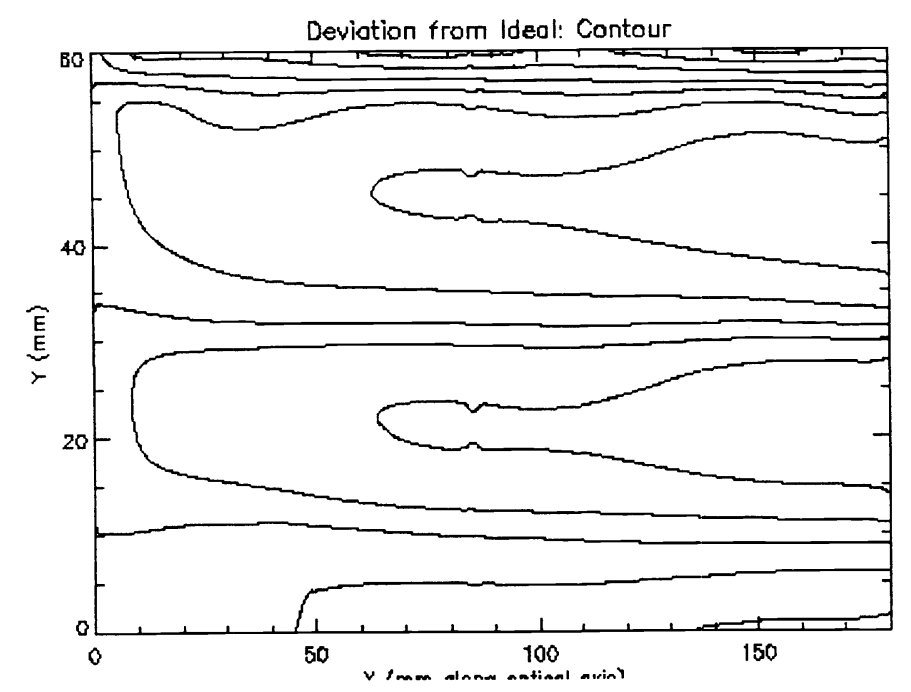

Figure 3: The contours of a thermally formed glass sample as measured on a Moore coordinate measuring machine. The figure shows the deviation from the ideal cylindrical form. The majority of piece has sub-arcminute figure.

\subsubsection{Mechanical Figure Measurements}

We have done considerable metrology on the formed glass pieces as the process has been developed over the past year. The Moore Tool Company precision metrology division performed the first sets of measurements while our internal metrology capability was being developed. Using an interferometrically controlled Moore CMM-10 coordinate measuring machine, a formed glass piece was scanned with a ruby tipped mechanical probe and the profile generated. The measurements were done both in low-force and zero-force mode and were repeated several times to ensure
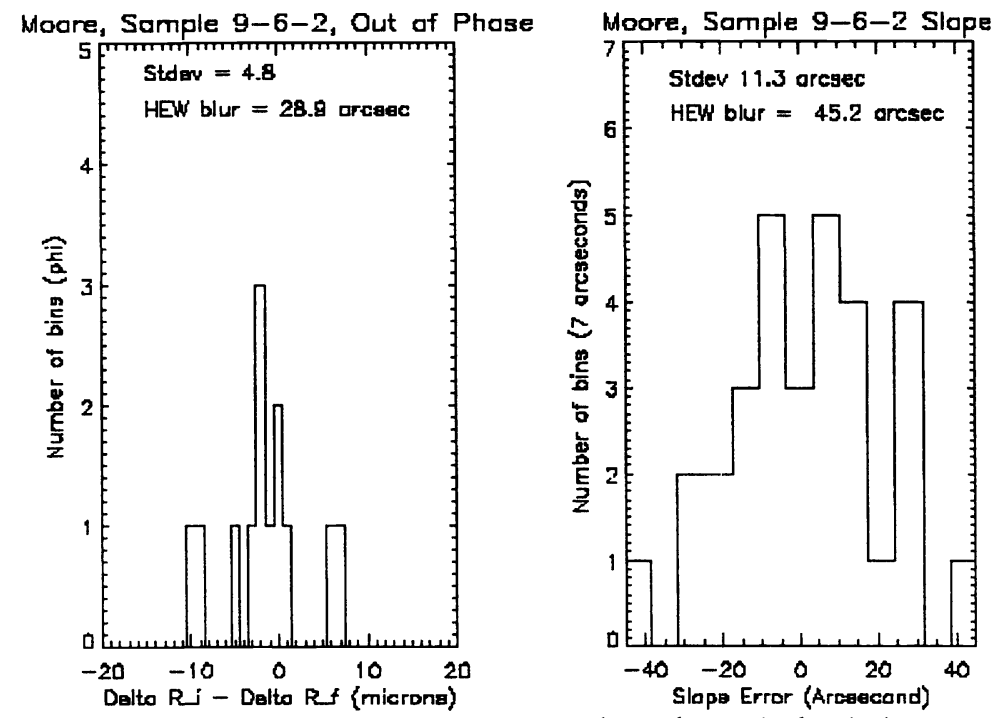

Figure 4: The data from a sample measured on the Moore CMM. The two dominant figure errors, out of phase roundness and slope are shown to be acceptable for HEFT performance goals.

accuracy and repeatability. Sixteen scans along the optical axis (at a step size of 100 microns) are combined to produce a surface profile of the pieces.

Figure 3 shows the actual contours of the surface of a representative piece, subtracted from that of an ideal cylinder. Figure 4 shows both the slope and out of phase roundness errors for the same sample. In both cases the HEW blur that would result from this piece, for a complete telescope constructed of these shells, is calculated. This 'system' HEW number, rather than individual shell figure errors, is the appropriate one for judging overall HEFT performance and is used throughout.

\subsubsection{Non-Contact Figure Measurements}

The large number of shells required by HEFT necessitates a rapid characterization and selection process. We have constructed $^{3}$ an apparatus to do this rapid selection (see paper by Jimenez-Garate et al., Conference 3444 ) and have characterized numerous samples. Axial scans performed confirm the results from the more involved Moore measurements and establish that we can routinely produce thermally formed glass pieces at the sub arcminute level. A representative set of scans is shown in Figure 5. 


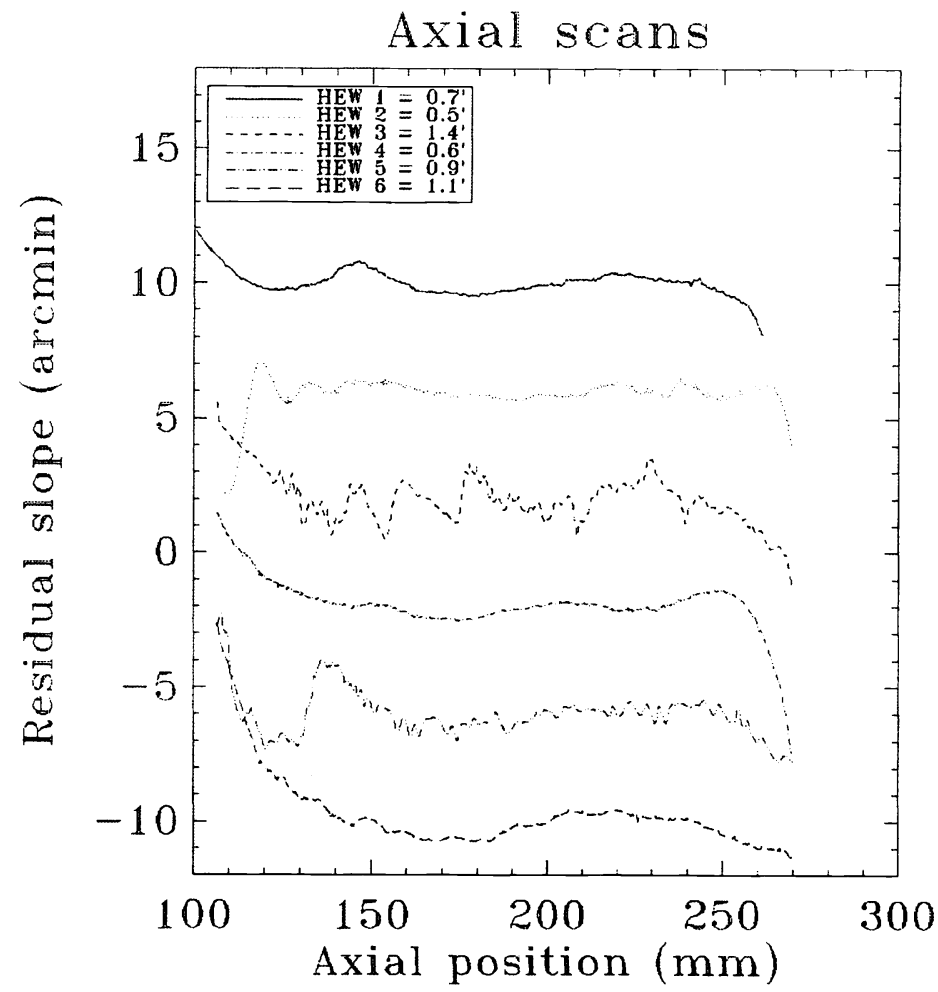

Figure 5: Laser scans of a representative sample of thermally formed glass pieces establish that the HEFT goals can be met with thermally formed glass. The average slope error is 36 arcseconds. The average contribution to overall system half energy width from this ensemble of samples is 52 arcseconds. Process improvement and tighter selection of samples can reduce the average contribution of the slope error to system performance to on order 30 arcseconds.

\subsubsection{Strength Measurements}

For the glass to work in practice, whether in a balloon-borne or satellite instrument, it must have sufficient strength to accommodate both the mounting stresses, and the stresses that the mirrors will see during a balloon flight profile or rocket launch. The overflow production method naturally produces glass of uncommonly high strength. The intrinsic defect size and rate is small. Dust or other particulate contamination does not contaminate the glass, reducing the chances of the glass developing flaws later (a dust grain can precipitate a flaw site). To ensure that the glass retains its strength through the thermal forming process, we conducted a number of strength trials using a standard double ring

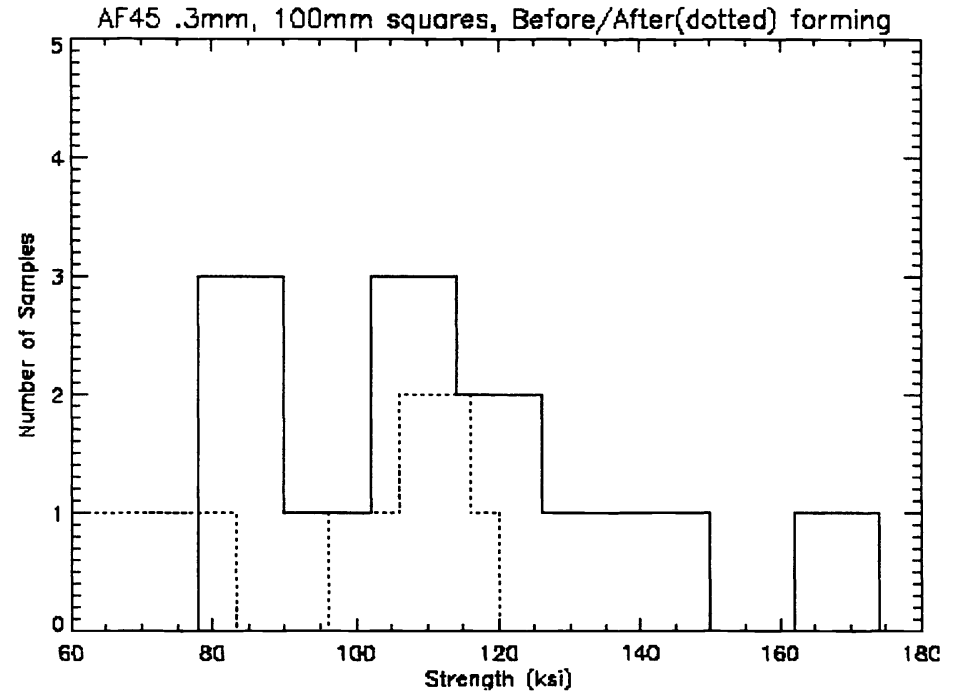

Figure 6: Strength tests on the glass, before and after thermal forming are consistent with little or no decrease in the glass properties during the forming process. 
method. Figure 6 shows that strength changes during the forming process are minimal. The glass maintains strength at least a factor of 6 above the value $(15 \mathrm{ksi})$ required by the HEFT mounting and mission profile parameters.

\section{COATING DEVELOPMENT}

The successful multilayer coating work reported ${ }^{4}$ by Mao et al (1997) has continued. A significant milestone was achieved by measuring the $\mathrm{x}$-ray performance of multilayer coated glass cylinders across a wide energy range. The fixed angle energy dispersive scans were performed at DSRI. A rotating anode $(\mathrm{Cu})$ source, capable of producing flux up to approximately $45 \mathrm{keV}$, was used to illuminate the samples. A Lithium drifted Germanium device was used as

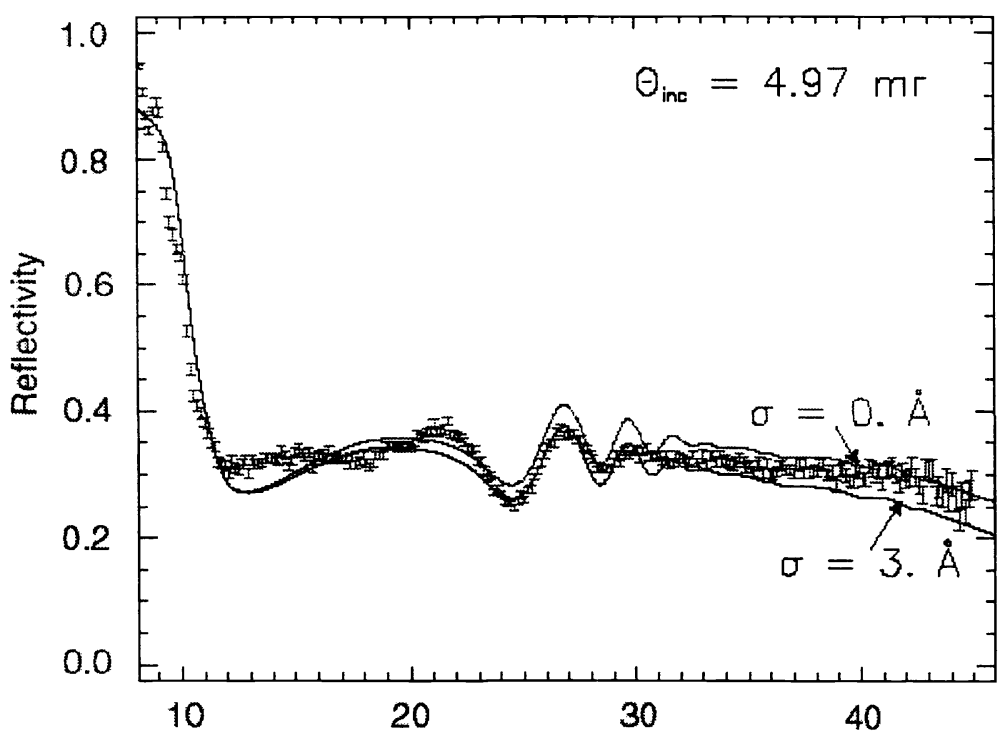

Figure 7: The X-ray performance of coated glass cylinders was measured across a wide energy band using DSRI facilities. The performance of the coating is consistent with a roughness of less than $3.5 \AA$

\section{Photon Energy [keV]}

the detector. The measurements were done at grazing incidence angles representative of the angles that the mirrors will be used at in the HEFT telescope. Figure 7 shows the data from a scan of a $10 \mathrm{~cm}$ radius glass cylinder coated with a $\mathrm{W} / \mathrm{Si}$ multilayer. The two fitted lines bound the roughness of the coating. The absolute calibration was not set for this scan, so we can only say that the measured performance exceeds that of the intended design with $3 \AA$ roughness. Other scans, where absolute calibration was done, demonstrate that $8 \mathrm{keV}$ specular results accurately predict the performance at higher energies.

Continuing work on optimization of the $\mathrm{M} / \mathrm{L}$ design for HEFT, along with success in reducing the roughness of the multilayers deposited on the glass substrates, has significantly increased the effective area of the experiment over the values originally proposed. Figure 8 shows the improved effective area that results from optimizing the coating design on the shells. In the design shown in Figure 8, W/Si is used on the outer shells and $\mathrm{Ni} / \mathrm{C}$ on the inner shells. We are also currently investigating designs based solely on $\mathrm{W} / \mathrm{Si}$.

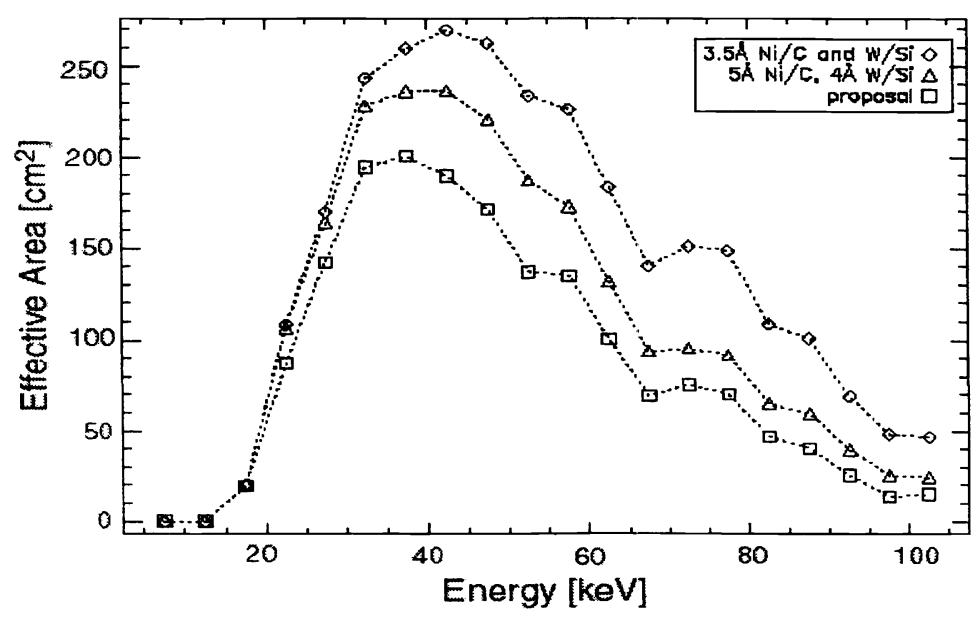

Figure 8: The effective area of the HEFT mission has increased by $40 \%$ over proposal values due to improvements in the design and the demonstrated roughness of the multilayer coating. 


\section{MOUNTING METHOD}

\subsection{Description of Method}

Our original concept ${ }^{1}$ for mounting the individual shells to make up the HEFT optic relied on a relatively conventional design using grooved spokes to constrain the shells into place on top and bottom. While this method is still viable, the small radius of the inner HEFT shells created problems with the groove spacing and did not pack these inner shells as efficiently as desired leading to loss of effective area at high energies. Investigation of alternate designs has led to the development of a novel mounting method that is currently being prototyped. Initial results (Section 4.2) are promising. The method relies on technologies and procedures developed during the production of the Reflection Grating Array for the X-ray Multi-Mirror observatory. A mechanical device, termed a sector base is the first unit in a mirror sector. Each sector begins with the production of a sector base to which glass shells will be mounted. This base is machined with the proper slope and radius for the first shell in the sector. Tungsten wires, of an appropriate diameter for the shell spacing desired, are epoxied (using a low outgassing, low shrinkage epoxy) to the mandrel. The assembly is then placed on an optically encoded mechanical grinder and the tungsten wires ground to conform to the desired position of the front of the next glass shell in the stack. The assembly is mounted in a fixture that easily rotates to present the evenly spaced tungsten wires to the grinding head. After grinding, the next shell is epoxied to the prepared tungsten surface and wires are attached to the back of this shell. After epoxy cure, the process is repeated. The grinding process is rapid and very accurate; errors do not 'stack up' as the sector is built. The metrology on the machines allows accuracy on order 1 micron (or 1 arcsecond over the $200 \mathrm{~mm}$ length of the HEFT shells). When producing a number of sectors, as required by HEFT, the manufacturing process is efficiently parallel. The number of shells that can be attached per day, 20-30 in a single shift, outstrips by a factor of two, the production of the multilayer coated shells. Each sector, once completed, is then assembled into a full optics module. The central mandrel is used as the base for attaching the sector to its neighbors in an exactly constrained fashion. The edges of the glass shells, which typically have poorer performance than the central $90 \%$, are trimmed before, or masked afterwards to eliminate broad wings in the telescope point spread function.

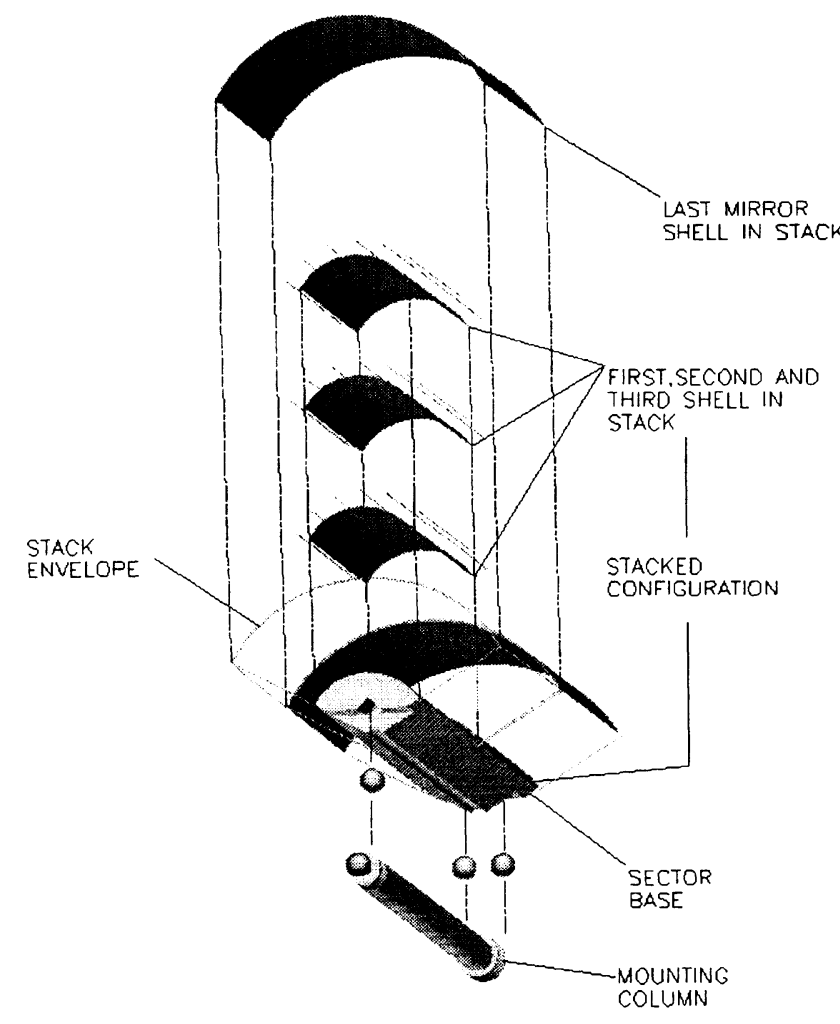

Figure 8: The mounting method being prototyped for the HEFT modules. The shells are assembled serially, epoxied to wires that serve as the spacing between shells as well as their support. The wires, once bonded, are ground to the proper conical approximation using an optically encoded grinding wheel. 


\subsection{Demonstration of Feasibility}

There are a number of issues to be addressed: 1) Do we get sufficient adhesion between metal and glass and/or coating to make the attachment robust? 2) Does the wire profile print through the glass and affect the optical surface? 3) How accurately can the entire stack-up be assembled? We have begun to address these issues by constructing prototype stacks. The first two issues were initially investigated by constructing flat stack ups using the Desag glass and tungsten wires. The resultant stacks are stiff and far more robust than the individual glass pieces. The adherence of the multilayer coating to the glass has been determined, using pull tests, to exceed that required by this mounting method To determine the effect of the wire/epoxy bond on the glass optics the flat glass pieces were traced by a Federal Surfanalyzer profilometer, before and after the wires were epoxied in place. The print through had amplitude of less

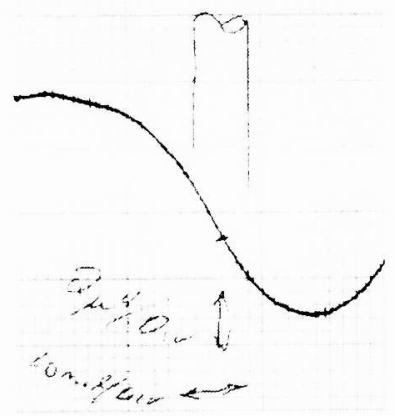

Figure 9: A profilometer scan over the region of a glass sample where a wire has been bonded shows no significant deviation induced by the wire bond line.

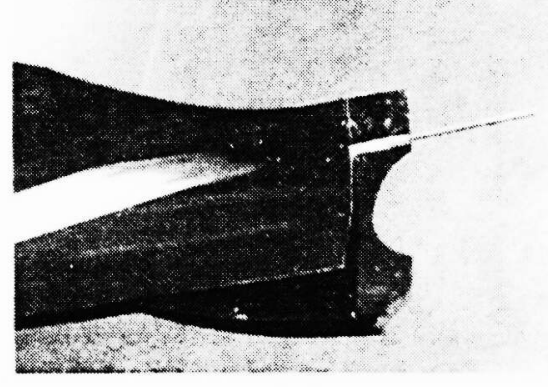

Figure 10: Bonding tests show that the epoxy adheres well to both the tungsten rod and multilayer coated formed glass.

than 0.1 microns through the 300 micron thick glass. At this level there is no measurable effect on the optics performance.

After demonstrating success with the flat stack-ups, we are now constructing cylindrical prototypes. For these units, we use a simplified base cylinder. The wires are spaced at 25 degrees around the perimeter. A Mylar sheet, with weights attached at each end, as shown in Figure 12, is used to equalize the pressure while the epoxy cures.

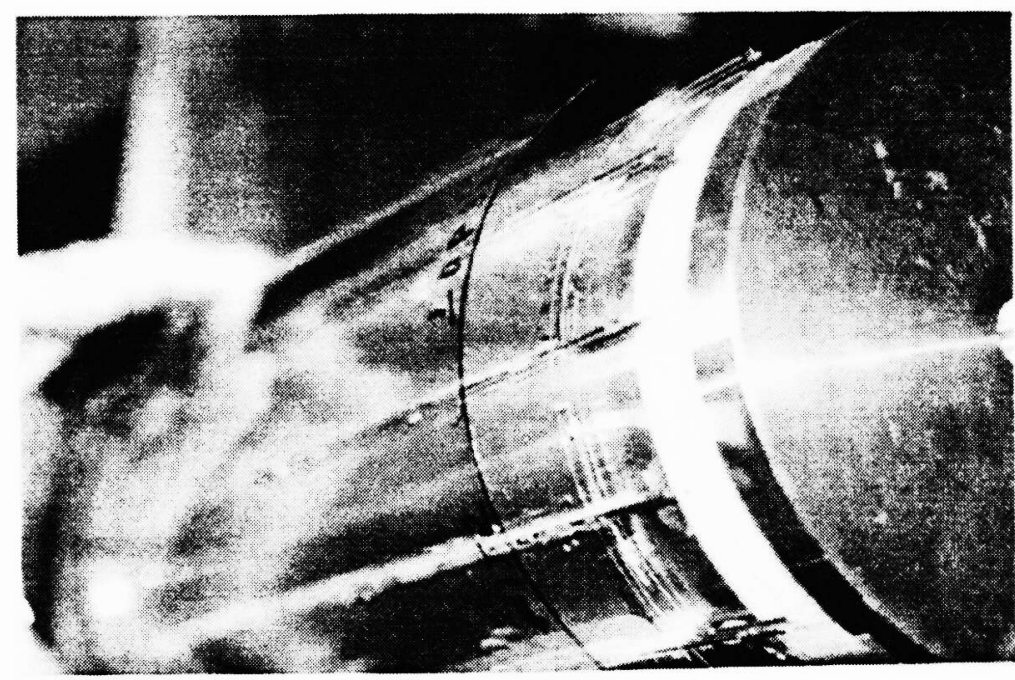

Figure 11: A glass shell being assembled onto the mounting prototype stack.

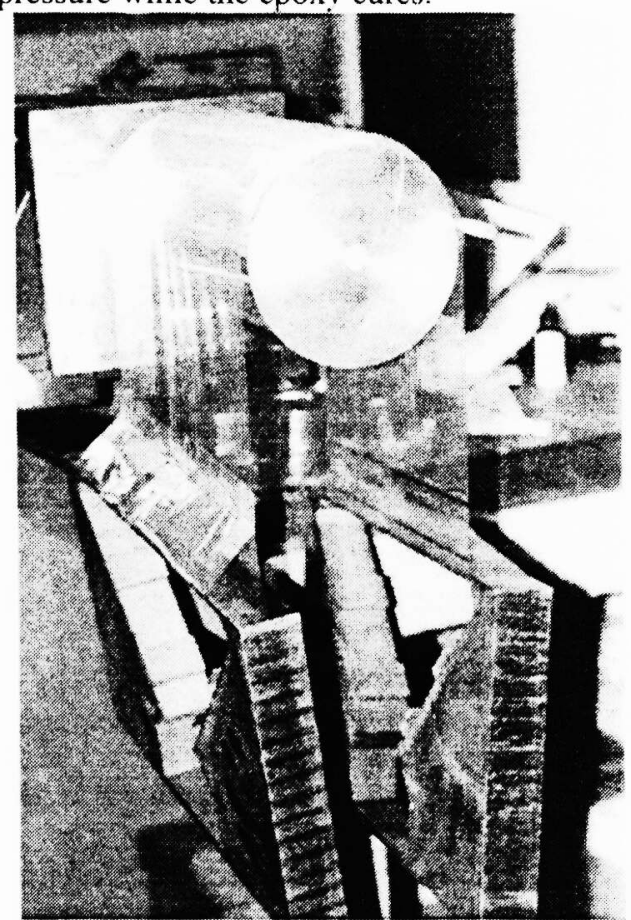

Figure 12: The glass stack has pressure applied during the epoxy cure to ensure uniform thickness of the epoxy layers and conformance between the glass and the bonding wires 
An edge on picture of a cylindrical stack is shown as Figure 13, the shells are uniform and are not deformed by the bond line. A full 25 shell mechanical stack is under construction at this writing. This stack will be characterized for figure and subjected to shake testing to validate the predicted survivability.

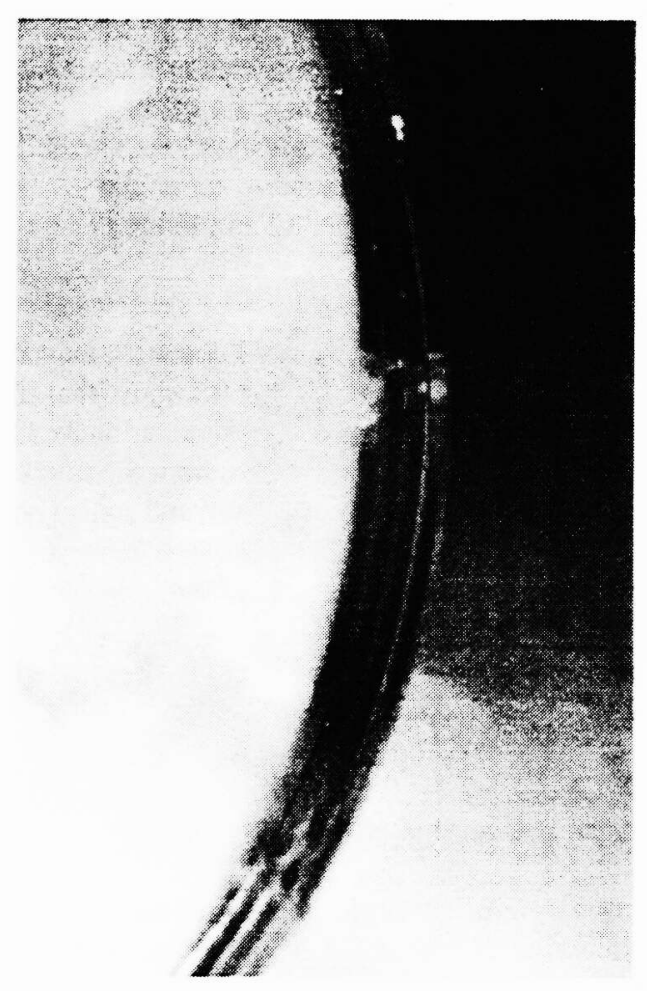

Figure 13: Close up, edge on view of a stack of 4 glass shells.

\section{CONCLUSION}

The thermally formed glass substrates adopted as the baseline for the optics on HEFT are now being produced with figure that results in better than 1 arcminute HEW for the HEFT telescopes. Refinements of the production method have increased performance and yield. A novel mounting method has been developed and prototyped. This mounting method, if successful, will produce very accurate, extremely strong mirror sectors that can readily be assembled into optics modules that meet the needs of HEFT and other hard X-ray telescope projects.

\section{ACKNOWLEDGEMENTS}

This work was supported under NASA grant NAG5-5129. M. Stern has helped to refine the thermal forming process and $\mathrm{K}$. Knox has helped quantify and study the process.

\section{REFERENCES}

1. C. J. Hailey et al., "Investigation of substrates and mounting techniques for the High Energy Focussing Telescope (HEFT)," SPIE Proc. 3114, pp. 535-543, EUV, X-Ray, and Gamma-Ray Instrumentation for Astronomy VIII, 1997

2. The HTXS mission study team, "The High Throughput X-ray Spectroscopy (HTXS) mission, the technology roadmap," a report to NASA, May 11, 1997.

3. Jimenez-Garate, M., Craig, W.W., Hailey, C.J. ., "Fast optical metrology of the hard X-ray optics for the High Energy Focusing Telescope", SPIE Proc. 3444, EUV, X-Ray, and Gamma-Ray Instrumentation for Astronomy IX, 1998.

4. P. H. Mao et al., "Development of grazing incidence multilayer mirrors for hard $\mathrm{x}$-ray focusing telescopes," SPIE Proc. 3114, pp. 526-534, EUV, X-Ray, and Gamma-Ray Instrumentation for Astronomy VIII, 1997. 